\title{
A simple new technique to measure the effective dead space of the face mask with a water volumeter in infants
}

\author{
M.G. Morris
}

\begin{abstract}
A simple new technique to measure the effective dead space of the face mask with a water volumeter in infants. M.G. Morris (C) ERS Journals Ltd 1999.

ABSTRACT: Measuring the effective dead space (EDS) of a face mask has been difficult in infants and the appropriate volume being deducted from lung volume measurements has varied between laboratories.

This study measured EDS in 16 infants (age range, 5-36 months) who have cystic fibrosis, undergoing lung volume measurement by $\mathbf{N}_{2}$ washout. A thin plastic bladder, whose neck resided in the mask port, was shaped to fill a size 1 clear face mask. A water volumeter was made by inserting the body of a $20 \mathrm{~mL}$ plastic syringe into the neck of the bladder forming a tight seal with a snug fit against the inner surface of the mask port. The mask was placed on a horizontal surface and water was added until a level appeared in the syringe body $\left(V_{1}\right)$. At end-inspiration, the mask was briefly placed on the mouth and nose of the sleeping infant, causing the water level to rise in the syringe body $\left(V_{2}\right)$. The actual total dead space $(V)$ of the mask when connected to the mouth port of the slide valve was $23 \mathrm{~mL}$. EDS $=V-\left(V_{2}-V_{1}\right)$.

Mean $(95 \%$ confidence interval $(95 \%$ CI $)$ ) EDS was 12.4 (95\% CI 11.2, 13.6) $\mathrm{mL}$. The smallest EDS was $8 \mathrm{~mL}$ since the connected ports (dead space, $8 \mathrm{~mL}$ ) were unlikely to be penetrated by the infant's nose or lips. EDS decreased with increasing body weight and height, but seemed to be influenced by individual facial features too.

In conclusion, a reliable noninvasive volumetric technique for the routine measurement of the effective dead space in infants has been developed.

Eur Respir J 1999; 14: 1163-1166.
\end{abstract}

Dept of Pediatrics, Pulmonary Medicine Section, University of Arkansas for Medical Sciences, Arkansas Children's Hospital, 800 Marshall Street, Little Rock, AR 72202-3591, USA. Fax: (501) 320-3930

Keywords: cystic fibrosis

lung volume

respiratory function tests in infants

Received: January 311999

Accepted after revision June 291999

This work was supported by a Clinical Research Grant (CG-008-N) cofunded by the American Lung Association (ALA) and the Arkansas Chapter of ALA. M.G. Morris was supported in part by the Department of Pediatrics, University of Arkansas for Medical Sciences.
While measuring the lung volume in infants has been regarded as physiologically and clinically important [1], accurate measurement is undoubtedly equally important. The application of a face mask to the face may significantly reduce the actual dead space (DS) of the mask [2]. Measuring the effective dead space (EDS) has been difficult and no methods have yet been published for use in infants [2]. Hence, the appropriate volume being deducted from lung volume measurements in infants has varied between laboratories [1-4].

The author has therefore developed a noninvasive technique to measure the EDS in infants. The aim of this study was to investigate the methodological aspects of the new technique and apply it to a group of infants undergoing lung volume measurement by $\mathrm{N}_{2}$ washout.

\section{Subjects and methods}

\section{Subjects}

Sixteen Caucasian infants and young children (7 males, 9 females) with a median age of 18.5 months (range, 5-36 months) who have cystic fibrosis and regularly attended the outpatient clinic of Arkansas Children's Hospital were studied during their routine infant lung function testing. The latter included lung volume measurement by the open circuit $\mathrm{N}_{2}$ washout technique using a commercial system, the Paediatric Pulmonary System 2600 (SensorMedics, Anaheim, CA, USA). Patients were excluded from lung function testing if they were clinically unstable, had a significant upper airway obstruction, or had had a recent thoracic or abdominal surgical procedure.

Patients were sedated with $80-100 \mathrm{mg} \cdot \mathrm{kg}$ body weight ${ }^{-1}$ chloral hydrate orally. The Infant Pulmonary Function Laboratory sedation policy and precautions were adopted. Patients were continuously monitored during lung function assessment with a Pulse Oximeter (Nellcor Inc., Pleasenton, CA, USA). The ethics committee of the institution approved the testing protocol. An informed written parental consent was obtained for each infant prior to testing and parents were present during tests.

Before the chloral hydrate was given, weight measurement was performed with a balance (Detecto, Webb City, MO, USA) that was checked for accuracy before each patient test. Weight was rounded to the nearest $20 \mathrm{~g}$. Two trained adults performed the stature measurement after the lung function test had been completed. A custom-made stadiometer was used, which had two, $91.5 \mathrm{~cm}$ long, steel rulers (Macklanburg-Duncan, Oklahoma City, OK, USA). They were mounted on the bottom and sideboards, exactly 
$20 \mathrm{~cm}$ from the headboard. Repeat measurements within $0.5 \mathrm{~cm}$ of each other were obtained. Measurements of length were made to the nearest millimetre [5]. EDS measurement was performed on the sleeping infant during the waiting period between $\mathrm{N}_{2}$ washouts.

\section{Methods}

A size 1 transparent face mask (Rendell-Baker Soucek Pediatric Face-mask; Gary Hull Anesthesia, Huntington Beach, CA, USA) was used. During the performance of $\mathrm{N}_{2}$ washout, the mask was connected to the mouth port (22 $\mathrm{mm}$ outer diameter, $15 \mathrm{~mm}$ inner diameter) of the pneumatic slide valve (Hans Rudolph Inc., Kansas City, MO, USA) that was used to switch the infant into pure oxygen. The connected mask and mouth port had a combined actual DS volume $(V)$ of $23 \mathrm{~mL}$.

\section{The water volumeter}

The body of a $20 \mathrm{~mL}$ plastic syringe (Becton Dickinson $\&$ Co., Franklin Lakes, NJ, USA) was used and the piston was discarded. The tip and conical end of the syringe body were cut off. A soft clear thin plastic bag $(10.16 \times 5.08 \times$ $30.48 \mathrm{~cm}$ ) (Tenneco Packaging, Specialty Products, Northbrook, IL, USA) was partially pulled through the port of a size 1 mask. A bladder, whose neck resided in the mask port, was shaped to fill the inside of the mask. Molding was facilitated by intermittently inflating the bladder with a gentle flow of air via a small cannula inserted through the bladder neck. The base of the bladder was confined to the mask space. The syringe body was inserted into the neck of the bladder forming a tight seal with a snug fit against the inner surface of the mask port. This leak-free unit made up of the syringe body, mask and bladder served as a water volumeter.

The mask was placed on a horizontal surface and water was added into the syringe body to fill the bladder until a water level could be seen in the syringe body. The bladder was squeezed repeatedly to get rid of air bubbles.

When laid on a horizontal surface, the plane of the mask curved slightly upwards. A baseline water level was obtained by holding the volumeter at a $45^{\circ}$ angle and placing the chin end of the mask on a clear sheet of thick plastic on a horizontal surface (fig. 1a). Then, the sheet edge was pulled up while the volumeter was simultaneously held firmly downward against the sheet and slowly tilted to place the mask against the sheet on the horizontal surface. Once the mask was horizontal (fig. 1b), a baseline reading of the water level in the syringe body was obtained $\left(V_{1}\right)$. A small piece of silicone putty was applied to the edge of the chin end of the mask to prevent the bladder from protruding beyond this edge.

Prior to using the volumeter on an infant, it was applied several times on a mannequin's face to establish the reproducibility of measurements, to test for leaks and more importantly to ensure that the setup was not likely to fall apart during mensuration. The sealed edge of the plastic bag was excluded from the bladder wall to further prevent possible bladder rupture. Furthermore, the inner $2 \mathrm{~mm}$ rim in the mask port situated at its junction with the dome of the mask was shaved in order to minimize resistance to water flow into the syringe body. Indeed, bladder rupture did not occur. a)

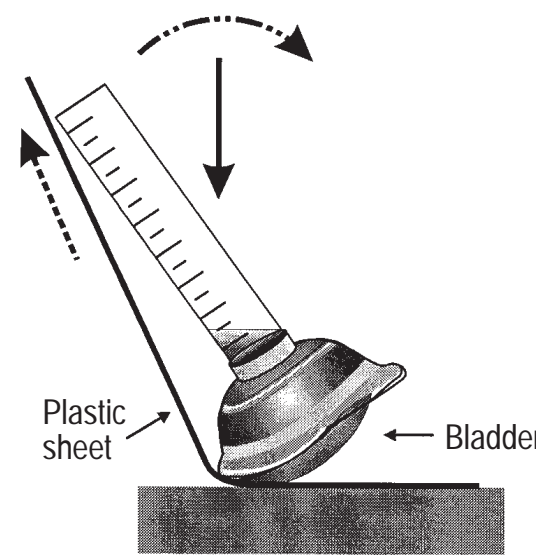

b)

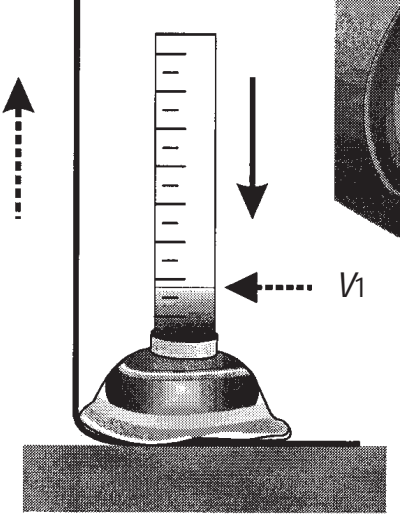

c)

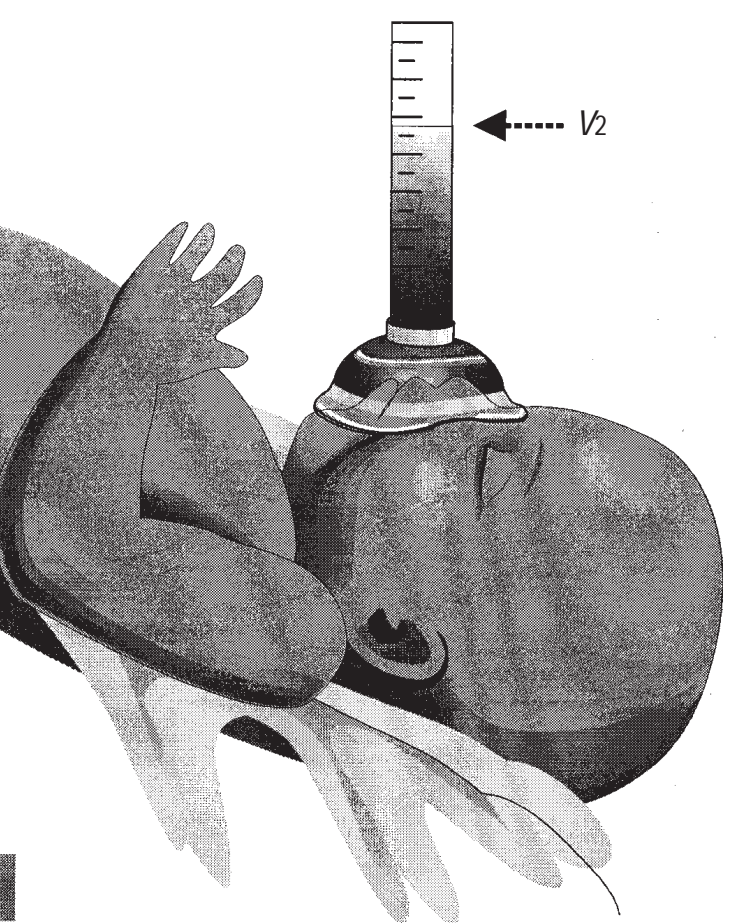

Fig. 1. - The water volumeter. A baseline water volume $(V 1)$ is obtained by holding the volumeter at a $45^{\circ}$ angle and placing the chin end of the mask on a clear sheet of thick plastic on a horizontal surface (a). Then, the sheet edge is pulled up (upward straight dotted arrow) while the volumeter is simultaneously held firmly downward (vertical solid arrow) against the sheet and slowly tilted (curved arrow) to place the mask against the sheet on the horizontal surface (b). At end-inspiration, the mask is quickly and briefly placed on the infant's nose and mouth that squeeze the bladder, displacing an equivalent volume of water $(V 2)$ into the syringe body $(\mathrm{c})$. 
The volumeter was held at a $45^{\circ}$ angle with one hand and the edge of the mask was placed against the chin of the sleeping infant, the other hand kept the infant's mouth closed by pushing the chin upwards. At end-inspiration the volumeter was quickly and briefly tilted to place the mask on the mouth and nose. This manoeuvre forced the water out of the bladder and into the syringe body (fig. 1c). This higher water level was recorded $\left(V_{2}\right)$. The bladder was not allowed to protrude beyond the edge of the mask. One operator performed the mask application on the infant's face and an assistant read and recorded the water level in the syringe body. Repeat measurements within $0.5 \mathrm{~mL}$ of each other were obtained. Assuming that the volume of displaced water was equal to the volume occupied within the mask by the facial tissues (nose, lips, and cheeks), then EDS $(\mathrm{mL})=V-\left(V_{2}-V_{1}\right)$ and substituting 23 for a size 1 mask $=23-\left(V_{2}-V_{1}\right)$

\section{Statistical analysis}

EDS was expressed as arithmetic mean and 95\% confidence interval $(95 \% \mathrm{CI})$. EDS from all patients were regressed against their respective age, height and weight measurements. The regression line and the 95\% CI were graphed. The coefficient of determination $\left(\mathrm{r}^{2}\right)$ was also computed. The slope and y-intercept of the regression equation were presented as mean and standard error of the mean (SEM). The unpaired t-test was used to test whether the slope was different from zero. The runs test was also performed to determine whether the data differ significantly from a straight line [6]. A p-value of $<0.05$ was regarded as significant.

\section{Results}

The group median weight was $10.3 \mathrm{~kg}$ (range, 5.8-17.3 $\mathrm{kg})$ and height, $80.4 \mathrm{~cm}(63.5-98.2 \mathrm{~cm})$. The mean effective dead space was 12.4 (95\% CI 11.2-13.6). EDS decreased with increasing weight $\left(\mathrm{r}^{2}=0.60\right)$ and height $\left(r^{2}=0.52\right)$ more than with age $\left(r^{2}=0.29\right)$ (fig. 2$)$. The slopes of the regression lines, were significantly non-zero $(\mathrm{p}=$ $0.0005,0.0017$ and 0.03 , respectively). Data were not significantly nonlinear $(\mathrm{p}=0.23)$, suggesting that they do follow a straight line rather than a curve [6].

\section{Discussion}

All infants remained clinically stable during EDS measurement. It took a very brief moment, probably 1-2 $\mathrm{s}$ to obtain a single reading. The technique appeared to be safe, was well tolerated by the infant and there was no oxygen desaturation. Given the very low resistance to water flow into the syringe body, bladder rupture did not occur.

The mean EDS was 12.4 (95\% CI 11.2-13.6). The minimum EDS in the present study was $8 \mathrm{~mL}$. The actual combined DS of the connected ports of the size 1 face mask and slide valve mouth port was $8 \mathrm{~mL}$. This clearly seemed to be an obligatory DS because it was unlikely for it to be penetrated by the infant's nose, lips or cheeks. Interestingly, it was found that the actual DS of the connected ports of size 0 or 2 face masks and the slide valve mouth port was also $8 \mathrm{~mL}$. It is thought that some previously reported EDSs in the literature were probably underestimated [3].

While EDS tended to decrease with increasing weight and height (fig. 2), it seemed that local facial characteristics, e.g. a small versus large nose, influenced the way the mask fitted on the infant's face, which in turn might have affected the measured EDS. Infants of different races may have varying amounts of dead space, depending on width of lips, nose, etc.

The described EDS was without any putty. If putty were to be utilized, then the actual DS of the mask ought to be measured first with the putty applied onto the mask edge. The bladder size will need to be confined to the remaining mask space if putty is placed within the mask to minimize the dead space. After the baseline reading is obtained, dead space measurements are then performed on the infant and EDS is calculated. The putty is then gently disconnected, without distorting its shape, from the mask of the volumeter. In order to maintain the identical mask dead space,
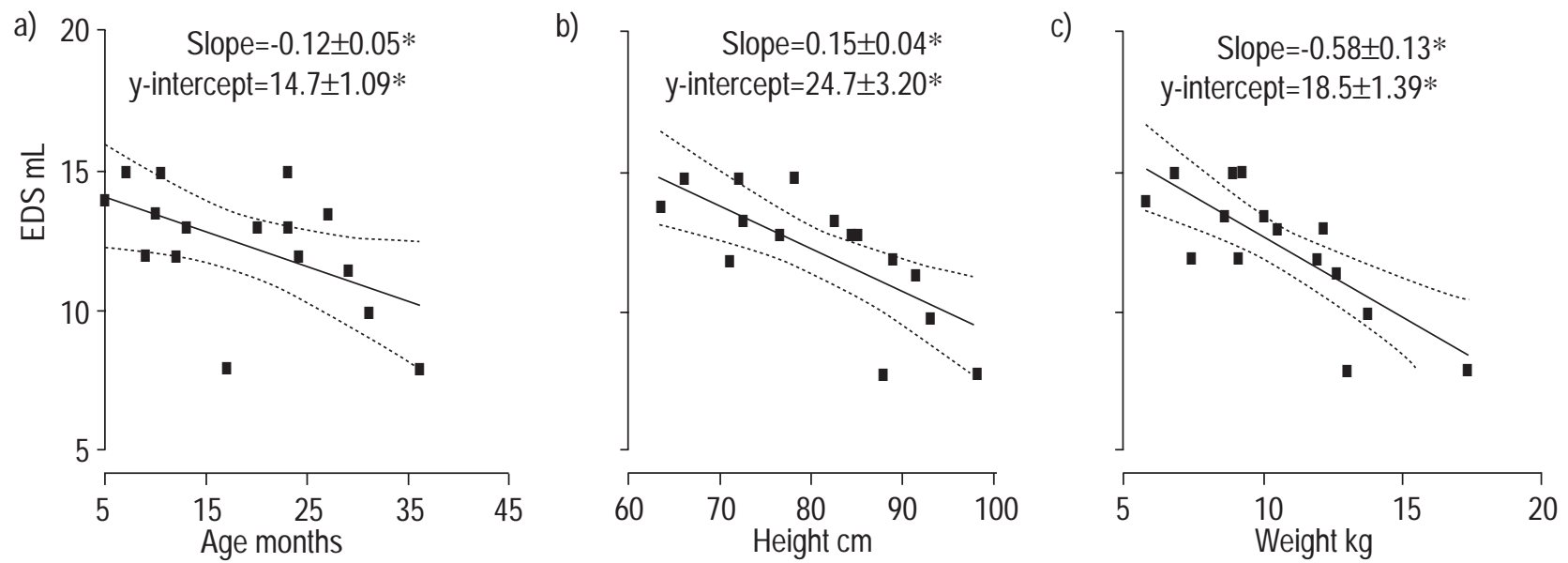

Fig. 2. - The effective dead space (EDS) ( $\bullet$ ) from each infant is plotted against the respective age (a), height (b) and weight (c). The straight solid line is the EDS regression line. The curved dotted lines represent the $95 \%$ confidence intervals. *: mean \pm SEM. 
another same size face mask, which will be utilized for lung volume measurement, is then embedded in the groove created by the edge of volumeter mask in this putty. The latter is then placed on the infant's face (M.G. Morris, unpublished data).

In conclusion, a simple, new and reliable technique has been developed to measure the effective dead space of the face mask with a water volumeter in infants. It is thought that the use of a standardized method to measure effective dead space would facilitate inter-laboratory comparisons of lung volumes in infants by reducing the variability in the appropriate volume being deducted from lung volume measurements.

\section{References}

1. American Thoracic Society/European Respiratory Society. Respiratory mechanics in infants: physiological evaluation in health and disease. Am Rev Respir Dis 1993; 147: 474-496.

2. Sly PD, Davis GM. Equipment requirement for infant respiratory function testing. In: Infant Respiratory Function Testing. Stocks J, Sly PD, Tepper RS, Morgan W eds. New York, John Wiley \& Sons Inc, 1996; pp. 45-8.

3. Gappa M, Fletcher ME, Dezateux CA, Stocks J. Comparison of nitrogen washout and plethysmographic measurements of lung volume in healthy infants. Am Rev Respir Dis 1993; 148: 1496-1501.

4. McCoy KS, Castile RG, Allen ED, Filbrun DA, Flucke RL, Bar-Yishay E. Functional residual capacity measurements by plethysmography and helium dilution in normal infants. Pediatr Pulmonol 1995; 19: 282-290.

5. Stocks J, Quanjer PhH. Reference values for residual volume, functional residual capacity and total lung capacity. Eur Respir J 1995; 8: 492-506.

6. Zar JH. Biostatistical analysis. 3rd Edn. New Jersey, Prentice Hall, 1996; pp. 317-370. 